\title{
Norois
}

Environnement, aménagement, société

$199 \mid 2006 / 2$

Canada : immigration, espaces urbanisés, parcs nationaux, loisir patrimonial

\section{L'immigration chinoise au Canada : logiques spatiales et nouvelles territorialités}

Chinese immigration in the Canadian: spatial distribution and new territorial strategies

Huhua Cao, Olivier Dehoorne et Vincent Roy

\section{OpenEdition}

Journals

\section{Édition électronique}

URL : https://journals.openedition.org/norois/1895

DOI : $10.4000 /$ norois. 1895

ISBN : 978-2-7535-1547-5

ISSN : $1760-8546$

Éditeur

Presses universitaires de Rennes

Édition imprimée

Date de publication : 1 juin 2006

Pagination : 11-22

ISBN : 978-2-7535-0332-8

ISSN : 0029-182X

Référence électronique

Huhua Cao, Olivier Dehoorne et Vincent Roy, «L'immigration chinoise au Canada : logiques spatiales et nouvelles territorialités ", Norois [En ligne], 199 | 2006/2, mis en ligne le 13 décembre 2008, consulté le 13 janvier 2022. URL : http://journals.openedition.org/norois/1895 ; DOl : https://doi.org/10.4000/ norois. 1895 


\title{
L'ImMigration CHINOISE AU CANADA :
}

\section{LOGIQUES SPATIALES ET NOUVELLES TERRITORIALITÉS}

\author{
Huhua CaO ${ }^{1}$, Olivier Dehoorne ${ }^{2}$, Vincent Roy ${ }^{1}$ \\ ${ }^{1}$ Département de géographie - Université d’OtTaWa (CANAda) \\ ${ }^{2}$ GEOdE-CARAibE (EA 929) - Université des Antilles et de la Guyane (Martinique - France) \\ caohuhua@uottawa.ca,olivier.dehoorne@martinique.univ-ag.fr,vroy013@uottawa.ca
}

\section{RÉSUMÉ}

La Chine est désormais le premier pays émetteur de migrants vers le Canada. Depuis les années 1990, les personnes de nationalité chinoise représentent près de $20 \%$ des nouveaux immigrants. Deux éléments fondamentaux permettent d'expliquer la nouvelle dimension de ces flux migratoires : tout d'abord l'abolition de l'accès préférentiel accordé aux natifs des pays européens (en 1967) et ensuite les politiques actuelles plus favorables à l'immigration (en moyenne 235000 nouveaux arrivants par an entre 1991 et 1995).

À travers ces flux migratoires renouvelés apparaissent de nouvelles stratégies territoriales: Vancouver et la Colombie-Britannique, les portes d'entrée historiques des immigrants asiatiques sur le sol canadien, sont désormais reléguées au second plan compte tenu du dynamisme de la région métropolitaine de Toronto.

Outre les considérations spatiales, il convient d'insister sur la perte de cohésion de cette communauté qui se scinde en sous-groupes, où les nouveaux arrivants se distinguent par des comportements plus individualistes. En effet les différences sociales, culturelles et économiques se précisent: les nouvelles générations plus diplômées sont mieux préparées pour réussir leur migration et s'insérer dans le tissu économique canadien. La diversité des origines géographiques des ressortissants issus du monde sinisé et le poids des événements historiques récents sont autant de paramètres à prendre en compte. Le fossé est important entre les premiers migrants originaires de l'ancienne colonie britannique de Hong Kong, les étudiants réfugiés de l'après-Tienanmen et les dernières générations de diplômés de la Chine de l'ouverture économique. Dans ce contexte, la Chinatown ne revêt plus la même valeur ni la même signification pour tous. Si les immigrants les moins favorisés, chinois et asiatiques en général, restent attachés à ce quartier historique, les nouveaux migrants aux statuts économiques plus confortables privilégient les quartiers résidentiels des banlieues nord-américaines.

Mots CLÉ : Canada - Chinatown - Chinois - Immigration - Migration - Stratégies territoriales.

ABSTRACT

Chinese immigration in the Canadian: spatial distribution and new territorial strategies

China is currently the principal source of immigrants coming to Canada. Since the 1990s, Chinese represent nearly 20 percent of new immigrants. Two fundamental pro- 
cesses provide an explanation for this new dimension in migration patterns: the abolition of preferential selection of migrants with European heritage (in 1967), and the pursuant policies that made immigration more acceptable in general (on average, Canada welcomed 235000 new immigrants per year between 1991 and 1995).

Accompanying these reinvigorated migratory fluxes are new territorial strategies: Vancouver and British Columbia, the historical ports of entry for Asian immigration on Canadian soil, are now relegated to second place due to the migratory pull of the dynamic metropolitan region of Toronto.

In addition to spatial considerations, it is important to highlight the loss of cohesion in this community that has divided into sub-groups, where the new arrivals distinguish themselves by their individual and differing behaviour. In effect, social, cultural and economic difference are pronounced: new generations are more educated, and thus, are better prepared to succeed and weave themselves into the Canadian economic fabric. The diversity of geographic origins of residents and the weight of recent historic events are also important parameters to take into account. The variance is significant between the first original immigrants from the former British colony of Hong Kong, the refugee students after the events of Tiananmen Square, and the latest generations of educated immigrants from China under open economy policies. In this context, Chinatown has neither the same value nor the same significance for all. If the immigrants, Chinese and Asians and general, who arrived under the discriminatory immigration policy, stay attached to this historic neighbourhood, the new immigrants with a more comfortable economic status will attach themselves to the residential neighbourhoods in the North American suburbs.

\section{KEY WORDS : Canada - Chinatown - Chinese - Immigration-Migration - Territorial Strategies.}

Pays d'immigration par excellence, le Canada compte en 2001 quelque 5,4 millions d'immigrants $^{1}$ (soit 18,2\% de la population totale) en provenance de toutes les régions du globe. Parmi eux, 2,1 millions de migrants arrivés après 1980 constituent ce qu'il est convenu d'appeler des immigrants récents ${ }^{2}$. Les personnes nées en Europe (principalement en Italie et au Royaume-Uni) représentent toujours la plus grande proportion des immigrants installés au Canada en 1996 mais leur part s'amenuise régulièrement (moins de la moitié de la population immigrante totale) face aux arrivées asiatiques (en 2001, $42 \%$ des immigrants sont originaires de sept pays asiatiques: Hong Kong, la Chine, l'Inde, les Philippines, le Sri Lanka et le Vietnam) et les deux pays les plus peuplés, la Chine et l'Inde, figurent au premier et deuxième rang des émetteurs vers le Canada : 124900 de nouveaux immigrants chinois sont arrivés au Canada entre 1996 et 2001.

Ces nouvelles tendances migratoires sont enregistrées dans un contexte de « boom » de l'immigration au Canada. En effet les politiques d'immigration ont changé au fil des ans et deux éléments fondamentaux doivent être soulignés : le premier, en 1967, fut l'abolition de l'accès préférentiel accordé aux natifs des pays européens et le second correspond à l'augmentation annuelle du nombre moyen d'immigrants admis qui est passé de 102000 , entre 1981 et 1985, à 235000 , entre 1991 et $1995^{3}$.

1. Population immigrante : personnes qui sont ou qui ont déjà été immigrants reçus. Les immigrants reçus sont des personnes qui ont obtenu des autorités de l'immigration le droit de résider au Canada en permanence. Certains immigrants résident au Canada depuis un certain nombre d'années, alors que d'autres sont arrivés depuis peu (Statistique Canada, 2001).

2. L'expression «immigrants récents » au sens strict qualifie officiellement les immigrants arrivés au cours de la dernière période intercensitaire. Lorsqu'on s'écarte des analyses purement statistiques, dans son acceptation la plus large, son emploi signifie les immigrants des deux dernières décennies.

3. De source officielle (Citoyenneté et Immigration Canada 2001), la politique canadienne d'immigration poursuit différents objectifs : répondre aux besoins du marché du travail, réunir les familles, respecter la tradition humanitaire. L'immigration est une responsabilité que le gouvernement fédéral partage avec les administrations provinciales; depuis la fin des années 
La problématique autour des immigrants chinois au Canada est particulièrement intéressante sur le plan quantitatif, puisque la Chine est devenue la principale source d'immigrants (Shi, 2004) depuis le milieu des années 1990 avec près de $20 \%$ du total des nouveaux immigrants (Statistique Canada, 2001), et également au regard de l'évolution des motivations des migrants : si l'image des Chinois quittant leur pays au début du XXe siècle en raison de la grande pauvreté et de l'instabilité politique demeure importante dans cette histoire migratoire, il faut souligner que les vagues migratoires suivantes ont été plus sélectives : ce furent des étudiants, des entrepreneurs et des investisseurs (Lai, 2003).

Dans le cadre de cette étude, nous commencerons par un bref historique des principales phases du fait migratoire chinois au Canada du XIX ${ }^{e}$ siècle jusqu'à nos jours pour ensuite analyser leurs logiques spatiales dans l'espace canadien; la plus grande diversité des stratégies territoriales permettra de caractériser l'évolution des profils des migrants.

\section{L'immigration chinoise au Canada : un regard sur l'histoire}

\section{L'ARRIVÉE DES PREMIERS IMMIGRANTS CHINOIS AU CANADA}

L'immigration chinoise vers le Canada a commencé au milieu du dix-neuvième siècle (Lai, 1988 ; Li, 1988; Wickberg 1984). Ces flux composés essentiellement d'hommes célibataires s’inscrivaient dans le prolongement de migrations déjà engagées en Asie du Sud-Est et dans les empires coloniaux en général. Les hommes fuyaient la misère d'un pays où manquait la terre dans un contexte de pressions démographiques exacerbées ${ }^{4}$. Aux défaites militaires et à l'instabilité politique s'ajoutaient des désastres naturels comme les inondations avec leur lot de famines.

Les processus migratoires étaient alors de deux ordres : l'émigration de coolies par « contrat » et l'émigration par étapes. Les coolies, qui étaient les plus démunis, s'engageaient par un « contrat » à travailler dans leur pays de destination pour rembourser l'intermédiaire qui avançait les frais de voyage. Ils pouvaient ensuite rechercher un emploi pour leur propre compte (Wickberg, 1984). La démarche est radicalement différente pour l'émigration par étapes où l'immigrant part travailler seul jusqu'à ce qu'il ait assez d'argent pour retourner en Chine; ensuite, à l'occasion de son premier retour au village natal, bien souvent il se marie et lors de son nouveau départ pour son pays d'exil un neveu ou un fils dans l'adolescence l'accompagne. Ainsi se constitue progressivement une filière migratoire. Selon sa situation financière et les possibilités du pays d'accueil, le migrant pourra éventuellement amener son épouse et reconstituer son foyer dans sa nouvelle patrie.

Les premiers immigrants chinois étaient normalement des coolies, engagés souvent en groupes pour accomplir des travaux de constructions ou d'extraction minière. Ensuite, après 1900, l'émigration par étapes fut la plus répandue au Canada (Wickberg, 1984).

La première vague d'immigrants chinois provenait des colonies britanniques ${ }^{5}$ et entra par la Colombie-Britannique, et plus particulièrement par l'île de Vancouver, en relation avec le mouvement de la Fraser gold rushes ${ }^{6}$. Les immigrants chinois venaient au Canada pour découvrir de l'or à la suite des rumeurs alimentées par les marchands chinois de San Francisco à propos des découvertes dans la Rivière Fraser. Cette situation a entraîné le déplacement d'immigrants chinois de la Californie à la Colombie-Britannique (Lai, 1988). La seconde vague migratoire en ColombieBritannique s'effectua lors des travaux du chemin de fer transcontinental à la fin des années 1870. Quelque 1500 Chinois expérimentés arrivèrent des États-Unis pour travailler à la construction du chemin de fer entre 1880 et 1881 (Lai, 1988; Wickberg, 1984). Au cours des quatre années de

1970, le gouvernement provincial se charge de concevoir et d'administrer certains aspects relatifs à la sélection et l'intégration des immigrants.

4. Alors que la population de la Chine a presque doublé en un siècle (410 millions d'habitants en 1850 contre 200 à 250 millions en 1750), la progression des terres cultivables est restée insuffisante (1210 millions mu en 1873 contre environ 950 millions mu (mou); quinze $m u$ (mou) équivaut à un hectare de terre) ( $\mathrm{Li}, 1988)$.

5. Il est à rappeler qu'au dix-neuvième siècle, le Canada était encore une colonie britannique.

6. Les précipitations d'or de Fraser (Rivière Fraser). 
grands travaux ferroviaires qui suivirent, le Canada reçut plus de dix-sept mille immigrants chinois dont dix mille en provenance directe de la Chine (Wickberg, 1984).

\section{UN SIÈCLE D'IMMIGRATION CHINOISE ENTRE RESTRICTION, EXCLUSION ET OUVERTURE PROGRESSIVE}

Au début du $\mathrm{XX}^{\mathrm{e}}$ siècle, environ deux mille immigrants chinois entraient en moyenne chaque année au Canada. La grande majorité demeurait concentrée en Colombie-Britannique et quelques-uns poursuivaient leur route vers l'Est canadien dès 1880. Les activités professionnelles se diversifiaient et les entreprises chinoises ne se limitaient plus à la satisfaction des seuls besoins de la communauté chinoise comme en atteste l'exemple des fameuses blanchisseries qui permettaient le déploiement des pionniers chinois à travers le territoire canadien. Dans la foulée, des Chinois ouvrirent des restaurants de style occidental, plus particulièrement dans la région des Prairies (Manitoba, Saskatchewan et Alberta). Mais dès les années 1890, les Chinois furent confrontés à une réduction constante des emplois accessibles face aux pressions de syndicalistes et d'hommes politiques : l'assemblée législative de la Colombie-Britannique légiféra pour interdire l'utilisation de la main-d'œuvre asiatique ${ }^{7}$. Le gouvernement et certains groupes de la province réclamaient d'Ottawa, à grand renfort de pétitions, des lois supprimant ou limitant sévèrement l'immigration asiatique. Cédant aux pressions, le gouvernement fédéral adopta une loi sur l'immigration chinoise qui entra en vigueur le $1^{\mathrm{er}}$ janvier $1902^{8}$. Comme celle qui l'avait précédée en 1885, cette loi stipulait qu'un navire ne pouvait transporter au Canada qu'un Chinois pour 50 tonnes de marchandises. Puis elle augmenta le droit d'entrée en le faisant passer de 50 à 100 dollars par personne. Enfin, en 1903, le Parlement du Canada adopta une nouvelle loi fixant le droit d'entrée de 500 dollars mais exemptant six catégories de personnes : les commerçants établis (une définition imprécise de cette catégorie a entraîné des abus), les diplomates, les ministres de culte, les touristes, les étudiants et les hommes de science (Wickberg, 1984).

Ces séries de restrictions à l'encontre les immigrants chinois n'ont pas eu d'effet significatif sur les arrivées comme en atteste la progression régulière des effectifs d'immigrants chinois en Colombie-Britannique ${ }^{9}$. Plusieurs d'entre eux quittèrent la province en train pour s'installer plus à l'Est. Cette diffusion favorisa l'émergence de puissants mouvements « anti-chinois » à travers tout le pays et conduisit en 1923 le gouvernement fédéral à adopter une loi d'exclusion interdisant aux Chinois l'accès au sol canadien. Durant cette période d'exclusion, de 1924-1947, seuls quelques Chinois furent autorisés à entrer au Canada (Lai, 1988) avec pour conséquence une chute de $25 \%$ des effectifs entre 1931 et 1941.

À la fin de la seconde guerre mondiale, la communauté chinoise fit porter ses efforts sur deux objectifs principaux : l'abrogation de la Loi sur l'immigration des Chinois (1923) et l'obtention du droit de vote pour les citoyens de la Colombie-Britannique d'origine chinoise (Wickberg, 1984). L'abrogation de la loi en mai 1947 mit effectivement un terme à un quart de siècle de régime d'exclusion. Ce fut une grande victoire morale sans être l'égalité rêvée. La Loi sur la citoyenneté canadienne, entrée en vigueur au début de 1947, fit du droit de vote une des prérogatives de la citoyenneté $^{10}$. La restriction de l'immigration chinoise au Canada entre 1947 et 1962 reflète le

7. Pour justifier cette dernière prohibition, on alléguait l'explosion de la mine de charbon de Nanaïmo en Colombie-Britannique dans laquelle 200 personnes avaient perdu la vie en 1887 à la responsabilité de travailleurs chinois (Wickberg, 1984).

8. Il est à noter que durant ces mêmes dates, la tendance était à l'exclusion des immigrants asiatiques en Amérique. D'ailleurs, la Chinese Exclusion Act, qui excluera les Chinois pour 10 ans, sera votée en 1882 et deviendra permanente en 1902 aux États-Unis.

9. Selon P. S. Li (1988), en 1881, la population chinoise au Canada était d'environ 4383 et elle s'élevait à 9129 en 1891. Entre 1891 et 1901, son effectif avait presque doublé, passant de 9129 personnes à 17312 , pour atteindre un total de 27831 individus dix ans plus tard.

10. D'après E. Wickberg (1984), il y a eu de nouveaux sympathisants pour la cause des chinois. De 1934 à 1946, le Parti CCF (Cooperative Commonwealth Federation) avait été à peu près la seule formation politique à exiger qu'on accorde sans aucune restriction, le droit de vote aux citoyens canadiens d'origine chinoise de la Colombie-Britannique. 
préjugé racial de la politique d'immigration canadienne contre les Asiatiques et autres immigrants non-blancs.

En 1962, le gouvernement du Canada changea les politiques pour les immigrants en créant quatre catégories d'admission ( $\mathrm{Li}, 1988)$ : les deux premières catégories attribuaient une immigration indépendante des gens professionnels et éduqués, la troisième catégorie était pour les immigrants ayant des relations privilégiées avec des citoyens canadiens ou des résidents permanents. Dans ces trois catégories, il n'y avait pas de référence de fait à l'origine raciale ni au pays d'origine pour l'admission du candidat. Toutefois, la quatrième catégorie d'admission permettait aux citoyens canadiens ou aux résidents permanents de parrainer des citoyens de n'importe quels pays d'Europe ou d'Amérique. Bref, les nouvelles politiques de 1962 permettaient aux Chinois sans lien de parenté au Canada de s'engager pour la première fois depuis 1923 comme immigrant indépendant ${ }^{11}$.

L'abrogation de la loi de 1923 marqua un renouveau pour les communautés chinoises canadiennes. Des changements conséquents dans la composition et les caractéristiques de la population chinoise apparurent une vingtaine d'années plus tard. Beaucoup de temps fut nécessaire pour que se dissipent les préjugés qui avaient inspiré la politique canadienne d'immigration et les derniers vestiges de la discrimination dont les Chinois avaient souffert ne tombèrent qu'en $1967^{12}$.

Pendant les années 1950 et 1960, la migration directe en provenance de la Chine continentale avait cessé; la plupart des Chinois immigrant au Canada étaient originaires de Hong Kong et quelques autres venaient des communautés chinoises d'outre-mer, de l'Asie du Sud-Est (Malaysia, Singapour et Philippines), du Sud de l'Afrique, d'Amérique latine (surtout du Pérou) et des Antilles. Rappelons qu'à cette période, le régime chinois contrôlait sévèrement les mouvements de ses citoyens et ce ne fût qu'à partir de 1974, sous le programme de la réunification familiale, que l'immigration chinoise pût recommencer (Liu, 1997). Au cours de la période de 1974 à 1988, la plupart des Chinois continentaux ${ }^{13}$ arrivaient au Canada pour rejoindre leur famille ou par l'entremise de leur réseau social. De plus, avec la réforme économique de Deng Xiaoping, entre 1978 et 1987, le gouvernement chinois envoya quelque 40000 étudiants étudier dans le système démocratique occidental et quelque 10000 étudiants supplémentaires les accompagnèrent par leurs propres moyens (Gittings, 1989). X.-F. Liu (1997) note à ce propos qu'à partir du début des années 1990, le niveau de scolarité des immigrants chinois de la République populaire de Chine était plus élevé que celui des autres immigrants arrivant au Canada ${ }^{14}$.

En l'espace d'un siècle les motivations des immigrants chinois entrant au Canada ont donc considérablement évolué : si la fuite de la pauvreté et la misère caractérisait les premiers migrants, à partir de 1967, les migrations sont nettement plus sélectives : elles concernent des étudiants, de personnes qualifiées, des entrepreneurs et d'hommes d'affaires à la recherche d'un environnement plus propice aux investissements et aux initiatives privées (Lai, 2003).

\section{Les immigrants chinois dans l'espace canadien}

L’immigration chinoise au Canada revêt donc une nouvelle dimension à partir des années 1980 compte tenu de l'abolition de l'accès préférentiel accordé aux Européens, de l'augmentation annuelle du nombre d'immigrants admis et des nouvelles libertés de déplacement accordées par

11. Depuis 1962, la seule catégorie d'immigrants ouverte aux chinois était celle de la parenté «sponsorisée » par des Sinocanadiens. Les immigrants des années 1950 étaient notamment des femmes et enfants qui furent séparés de leur mari et/ou père durant la longue période d'exclusion.

12. Selon E. Wickberg (1984), l'année 1967 fut importante non seulement à cause des modifications apportées au règlement canadien sur l'immigration mais aussi en raison de la Révolution culturelle chinoise qui, débordant la province de Guangdong, se répandit à Hong Kong.

13. Le terme « chinois continentaux » réfère à tous les immigrants provenant de la Chine continentale par opposition à ceux d'Hong Kong et de Taïwan.

14. Il est important de noter que les immigrants de la Chine continentale admis au Canada durant cette période et sous la politique canadienne relative à l'incident du Tiananmen Square de 1989 furent admis comme étant des immigrants indépendants. 
la Chine à ses ressortissants. La progressive diffusion spatiale observée depuis le début du XXe siècle se précise : si la ville de Vancouver porte d'entrée historique des Chinois au Canada reste une place essentielle, de nouvelles stratégies se dessinent.

Le tableau 1 permet de suivre la distribution spatiale des immigrants chinois au Canada de 1961 à 2001 en insistant sur les positions primordiales des 32 grands centres urbains (GCU). Les immigrants chinois constituent d'abord une population urbaine qui se concentre dans les métropoles les plus dynamiques. Dans ces conditions, $97 \%$ des immigrants chinois se trouvent dans ces 32 (GCU) (fig. 1).

\begin{tabular}{|c|c|c|c|}
\hline & Canada & 32 GCU & $\%$ \\
\hline Avant 1961 & 15850 & 14550 & $91,80 \%$ \\
\hline $1961-1970$ & 18500 & 17745 & $95,92 \%$ \\
\hline $1971-1980$ & 38805 & 37485 & $96,61 \%$ \\
\hline $1981-1990$ & 62315 & 60845 & $97,64 \%$ \\
\hline $1991-2000$ & 197355 & 193515 & $98,05 \%$ \\
\hline Total & 332825 & 324135 & $97,40 \%$ \\
\hline
\end{tabular}

Tableau 1 : Les nouveaux immigrants de la République populaire de Chine au Canada (Source : Statistique Canada, 2001)

The New Immigrants of the People's Republic of China in Canada

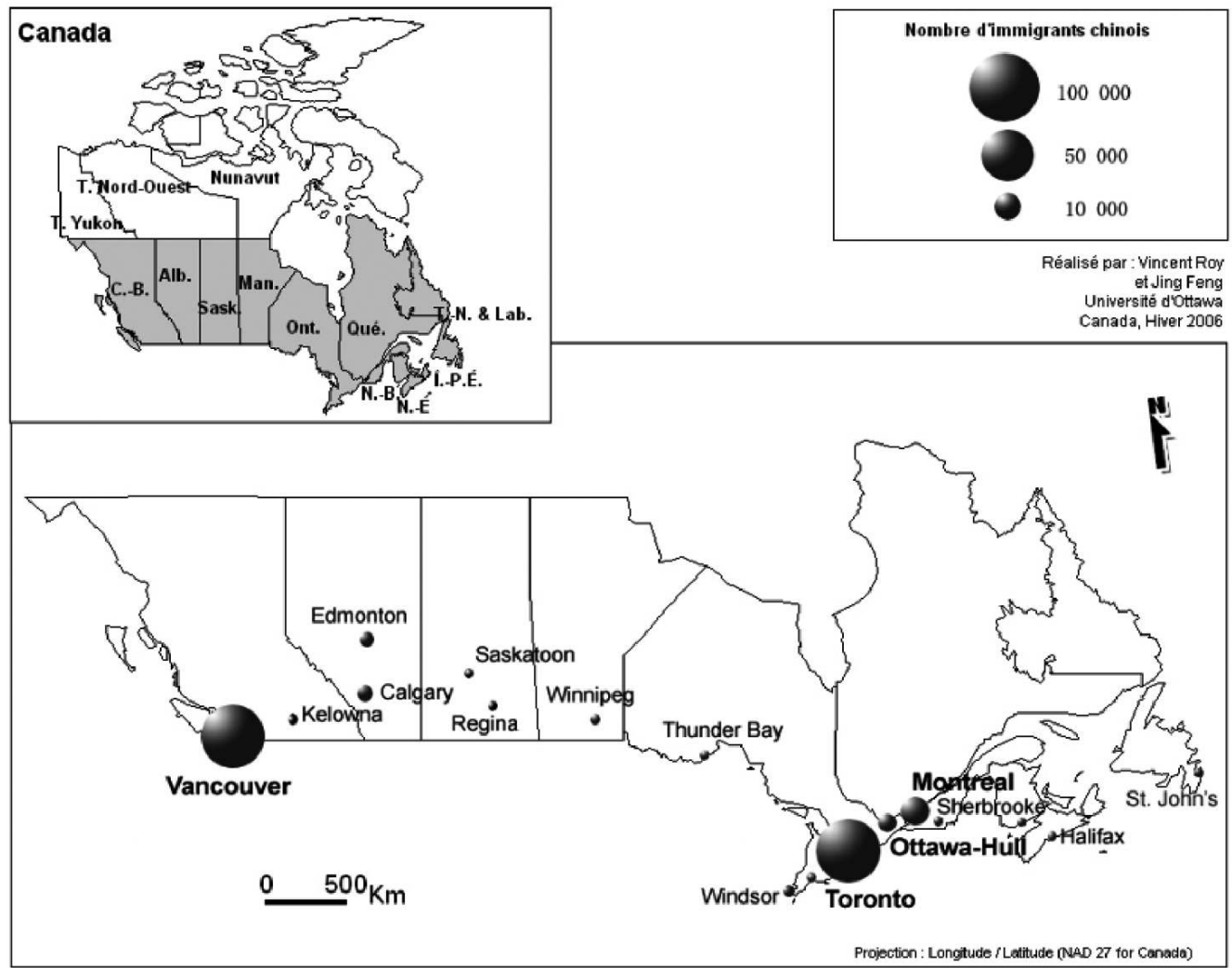

Figure 1 : Répartition spatiale d'immigrants chinois dans 32 Grands centres urbains au Canada, 2001 (Source : Statistique Canada, 2001)

Spatial Distribution of Chinese Immigrants in the 32 Large Urban Centres of Canada, 2001 
Selon les données disponibles, il apparaît que les immigrants chinois privilégient d'abord les trois principaux GCU canadiens : Toronto, Vancouver et Montréal. Leur concentration s'y est renforcée au fil des recensements : désormais $78 \%$ des migrants y résident contre $65 \%$ en 1961 . Toutefois la situation de Vancouver est plus nuancée car depuis 1975 elle a perdu progressivement son statut de la première ville d'accueil pour les immigrants chinois. Vancouver regroupe actuellement $31 \%$ de ces migrants, soit 4 points de moins que dans les années 1970. Soulignons que cela n'empêche pas l'augmentation de ces effectifs en nombre absolu avec soit 34445 arrivants entre 1996-2001 contre 24055 entre 1991-1995. La région métropolitaine de Toronto figure en tête avec $43 \%$ du total des nouveaux immigrants chinois ce qui correspond à 54930 personnes supplémentaires lors de la dernière période intercensitaire et 30415 entre 1991 et 1995 . Toronto est désormais le premier lieu d'accueil et de résidence des nouveaux migrants. La région urbaine de Montréal reste bien en retrait avec un peu plus de $6 \%$ des migrants chinois.

Compte tenu de la concentration en milieu urbain des migrants, les résultats notés au niveau des GCU sont corrélés par les situations observables dans les différentes provinces. Ainsi la province de la Colombie-Britannique qui était la principale zone d'accueil est dorénavant devancée par l'Ontario qui a enregistré un doublement des arrivées des nouveaux immigrants chinois lors des deux dernières périodes intercensitaires : la région a accueilli 68795 personnes entre 1996 et 2001, soit presque 25000 de plus que la Colombie-Britannique (fig. 2). À l'image de la province et de Vancouver, la ville de Victoria n'enregistre plus que $1 \%$ des immigrants chinois (soit 3170 personnes) contre $3 \%$ en 1961.

Figure 2 : Évolution des immigrants chinois dans les provinces canadiennes de 1961 à 2001 (Source : Statistique Canada, 2001)

Chinese Immigration in the Canadian Provinces, 1961-2001

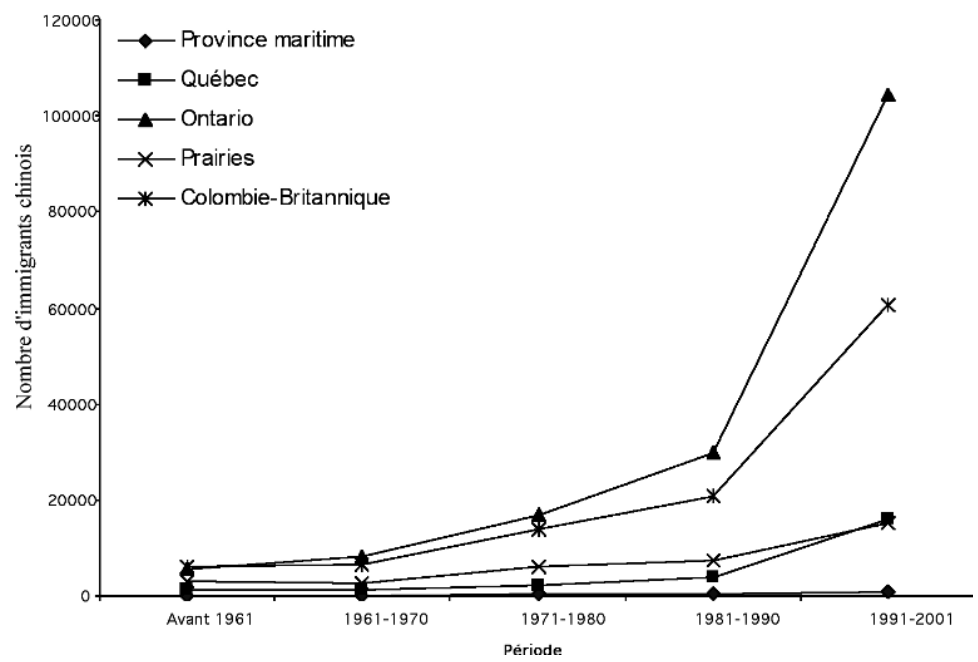

Les flux d'arrivants des dernières décennies attestent d'une nouvelle polarisation des immigrants chinois au profit de l'Ontario, de sa capitale économique Toronto évidemment mais aussi plus largement de l'ensemble de sa région métropolitaine. Par exemple les villes de Kitchener et Windsor se distinguent par de remarquables croissances ces dernières années. À Kitchener, le nombre de nouveaux migrants est ainsi passé de 320 entre 1991 et 1995 à 1355 cinq ans plus tard. Les villes de Guelph et Kingston ont accueilli plus de 400 nouveaux immigrants chinois entre 1996 et 2001 contre moins d'une centaine cinq ans auparavant. L'augmentation la plus significative concerne la ville Ottawa-Hull avec plus de 7000 arrivées entre 1996 et 2001, soit un triplement entre les deux périodes intercensitaires. Elle accueille désormais $4 \%$ de ces migrants. Incontestablement la polarisation exercée par Toronto au niveau national profite à l'ensemble du réseau urbain de la province. Un maillage plus serré se met place autour de Toronto et se prolonge par un corridor 
urbain qui s'étend jusqu’à Montréal. Le dynamisme économique de la région, notamment dans le domaine des nouvelles technologies, est décisif.

La région des Prairies (composée de trois provinces, soit l'Alberta, la Saskatchewan ainsi que le Manitoba et de 5 GCU) connaît aussi des tendances similaires à celle de la Colombie-britannique mais avec des flux bien inférieurs en valeur absolue. La part relative des nouveaux immigrants chinois y diminue tandis que ces derniers à l'échelle de ces provinces se concentrent dans les cinq régions métropolitaines (essentiellement Calgary et Edmonton) à plus de $93 \%$. Dans la province du Québec et la région de l'Atlantique (Nouveau-Brunswick, Nouvelle-Écosse, Terre-Neuve et 4 GCU), la modeste progression des arrivées en valeur se traduit également par une régression de leur part respective à l'échelle du Canada (fig. 3). En fait l'ensemble de ces régions fait l'objet d'un maillage territorial relativement lâche de la part des migrants chinois. Ces migrants aux activités moins diversifiées travaillent notamment dans des secteurs traditionnels comme celui de la restauration. La présence des immigrants est en quelque sorte proportionnelle au potentiel économique de ces régions. Ces espaces périphériques peuvent aussi constituer des étapes qui s'inscrivent dans une stratégie migratoire individuelle : des nouveaux immigrants indépendants y font leur première expérience professionnelle avant de poursuivre leur trajectoire individuelle vers les centres économiques du pays.

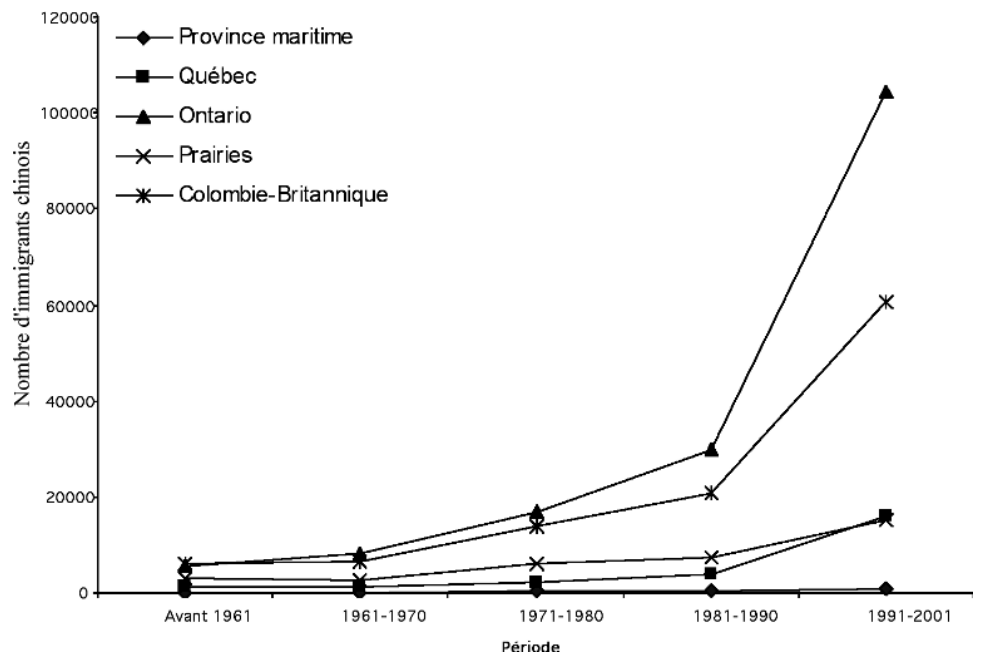

Figure 3 : Évolution d'immigrants chinois des Grands centres urbains dans les cinq régions au Canada, 19612001 (Source : Statistique Canada, 2001)

Regional Evolution of Chinese Immigrants in Canada's Large Urban Centres, 1961-2001

\section{Entre Chinatown et banlieues, des communautés immigrées plurielles}

\section{UN NOUVEAU REGARD SUR LA CHINATOWN}

La Chinatown, dont la formation a d'abord consisté à la construction d'une enclave chinoise distincte, géographiquement et culturellement, du reste de la population, face à la discrimination dont elle faisait l'objet de la part de la société canadienne, est aussi au cœur d'un processus d'assimilation des Chinois nés au Canada et de leur adaptation à la société d'accueil. Le renouveau de l'immigration chinoise à la fin des années 1960, accompagné d'investissements, a permis la revitalisation des anciennes Chinatowns et contribué au développement de nouveaux Chinatowns (Lai, 2003) et plus généralement à l'ouverture des centres d'achats chinois ou asiatiques en banlieue, notamment pour les régions métropolitaines de Toronto et Vancouver. Ces nouveaux projets de développement sont possibles grâce aux capitaux chinois provenant de la Chine qui constituent un élément important du transnationalisme. Le quartier chinois est devenu le centre 
culturel symbolique et commercial de la communauté chinoise mais il n'est plus la destination incontournable des nouveaux immigrants ( $\mathrm{Li}, 2000)$.

La suburbanisation est le phénomène récent qui caractérise les nouvelles logiques territoriales des immigrants chinois. Comme étudiée par D. Fang et D. Brown (1999), à partir de trois grandes villes étasuniennes (New York, Los Angeles et San Francisco), la mobilité spatiale des immigrants chinois s'étend aux banlieues longtemps considérées comme des quartiers ethniques et raciaux homogènes à classe sociale élevée. Les Chinois ont tendance à quitter des régions à forte concentration ethnique pour s'installer dans les secteurs où les opportunités d'emploi sont meilleures. Ainsi il apparaît que les Chinois habitant dans les banlieues ont un statut socio-économique plus élevé que les résidents chinois du centre-ville davantage portés à vivre au sein des communautés chinoises. Les résidents des Chinatowns, souvent plus âgés, peu diplômés et plus limités dans leurs aptitudes professionnelles, se différencient des immigrants chinois plus jeunes caractérisés par une mobilité géographique plus flexible, une meilleure éducation et de plus grandes facilités en anglais.

Au cours des deux dernières décennies (soit entre les recensements de 1981 et 2001), le nombre de quartiers ethniques ${ }^{15}$ des trois grandes villes canadiennes est passé de 6 à 254 et un peu plus de $60 \%$ de ces quartiers sont chinois (Keung, 2004). À Toronto, N. Keung (2004) souligne que la plupart des quartiers chinois, contrairement au passé, se situent principalement en banlieue; moins de $10 \%$ de ceux-ci se localisant au centre-ville. Les immigrants chinois s'établissent donc dans certaines banlieues de Toronto grâce à l'épanouissement du secteur des haute-technologies mieux développé et aux meilleures opportunités d'emploi. Néanmoins un rapport de Statistiques Canada, publié en 2004, stipule que les quartiers de minorités visibles ${ }^{16}$ restent affectés par des taux de chômage plus élevés et des niveaux de revenu plus faibles que la moyenne nationale.

En fait la comparaison entre les profils des Chinois installés à l'intérieur d'une enclave et ceux de l'extérieur laisse apparaître une nette différenciation de leur statut socio-économique : l'opposition sociale entre le centre-ville et les banlieues est bien marquée comme l'observent L. Lo et S. Wang (1997) à Toronto. Les Chinois qui habitent la banlieue, particulièrement depuis le milieu des années 1980, jouissent d'un meilleur capital humain et proviennent notamment de Hong Kong ou Taïwan. Ce sont des immigrants indépendants, investisseurs et hommes d'affaires. À l'inverse les Chinois qui habitent le centre-ville de Toronto sont plutôt d'origine chinoise ou vietnamienne. Ces migrants plus âgés, réfugiés ou venus dans le cadre d'un rapprochement familial, souffrent du plus haut taux de chômage.

\section{OTTAWA, UN EXEMPLE SINGULIER RÉVÉLATEUR DU NOUVEAU PROFIL DES IMMIGRANTS CHINOIS}

Au cours de la dernière décennie, un peu plus de 7000 nouveaux immigrants chinois se sont installés à Ottawa, soit un effectif qui correspond à $40 \%$ de cette population immigrante totale. La ville compte désormais un immigrant pour cinq résidents et une personne sur cinq appartient à une minorité visible. Et un doublement est prévu d'ici les deux prochaines décennies. La communauté chinoise, avec plus de 30000 membres (Statistique Canada, 2001), correspond au groupe de minorité visible le plus important à Ottawa (O’Neill, 2003). Si les premiers Chinois arrivés à Ottawa avaient immigré depuis la Colombie-Britannique lors de l'achèvement du réseau ferroviaire, vers la fin du XIX ${ }^{\mathrm{e}}$ siècle $(\mathrm{Li}, 2000)$, la croissance de la population chinoise d'Ottawa est très récente. La

15. « De 1981 à 2001, les quartiers de minorités visibles dans les trois principales régions métropolitaines du Canada ont connu une expansion. La recherche dans le domaine laisse entendre que les minorités ethniques se regroupent en enclaves pour des raisons de liens familiaux et communautaires. Les nouveaux immigrants sont peut-être aussi restreints aux quartiers pauvres où se trouvent des logements abordables étant donné qu'ils se situent généralement au bas de l'échelle socio-économique dans leur nouvelle collectivité » (Infrastructure Canada, 2005).

16. «Les quartiers de minorités visibles, définis comme les secteurs de recensement dont plus de $30 \%$ de la population appartient à une même minorité visible, se sont multipliés de 1981 à 2001 dans les trois principales régions métropolitaines du pays, leur nombre passant de 6 à 254 » (Statistique Canada, 2004). 
Huhua Cao, Olivier Dehoorne, Vincent Roy

ville comptait tout juste 1000 Chinois au début des années 1960 puis 3800 en 1981 et 7600 en 1991. Comparativement aux autres Chinatowns canadiens, la Chinatown d'Ottawa possède une courte histoire dont la croissance est davantage liée à l'arrivée soudaine de plus de 4000 réfugiés d'Indochine qui ont développé des restaurants vietnamiens et d'épiceries ( $\mathrm{Li}, 2000)$.

L'essor de l'immigration chinoise s'est précisé à partir du milieu des années 1990 avec près de 20000 immigrants chinois à la date de 1996. Ce sont les perspectives du secteur de la haute-technologie qui attirent les immigrants chinois, notamment des ingénieurs et programmeurs chinois diplômés à l'image de la municipalité de Kanata dans la banlieue d'Ottawa où sont implantées plusieurs grandes entreprises spécialisées comme Nortel; cette dernière entreprise a engagé plus de 10000 immigrants chinois lors des dernières décennies. La communauté chinoise de Kanata s'est ensuite structurée avec l'ouverture de magasins chinois/alimentaires spécialisés et la création de petites entreprises chinoises de sous-traitance performantes. Kanata est ainsi devenu l'un des lieux d’installation privilégiés des nouveaux immigrants chinois.

Les premières vagues d'immigrants chinois d'Ottawa provenaient soit de Hong Kong ou Taiwan, des territoires chinois beaucoup plus occidentalisés à la République populaire de Chine beaucoup plus occidentalisés et orientés vers les affaires, et ceux-ci parlaient le cantonais et l'anglais et étaient souvent de croyance chrétienne. Ces immigrants ont créé des commerces comme les restaurants, des épiceries et les buanderies. À l'inverse les nouveaux flux migratoires correspondent à des Chinois non-croyants, plus qualifiés comme des docteurs, des ingénieurs et des professeurs provenant de la Chine continentale, une société beaucoup plus traditionnelle qui parle majoritairement le mandarin. La langue des affaires de la communauté chinoise d'Ottawa est donc aujourd'hui le mandarin.

Selon leur culture, leur histoire, leur origine géographique, leur statut socio-économique, les modes de vie et les lieux de vie des immigrants regroupés sous le qualificatif commun de « chinois » varient donc considérablement. Ces réalités mettent en évidence une communauté plurielle, des communautés multiples où les stratégies individuelles prennent de plus en plus d'importance dans les trajectoires migratoires. Par conséquent, à l'échelle de la région métropolitaine d'Ottawa-Hull, les immigrants chinois sont davantage dispersés que toute autre ville canadienne ${ }^{17}$. La plupart des Chinois d'Ottawa ne sont pas ségrégués spatialement et n'habitent donc pas la Chinatown d'Ottawa. Ces derniers privilégient les nouveaux pavillons situés en banlieue au détriment des plus anciens logements du quartier chinois et la vie de banlieue semble être l'alternative idéale dans leur processus d'intégration. Le processus de suburbanisation, particulièrement important vers le début des années 1980 a contribué à limiter la croissance de la Chinatown d'Ottawa.

Plus que jamais les immigrants chinois anciens et nouveaux forment bien une communauté plurielle où les différences sociales, culturelles et économiques sont importantes. Les nouvelles générations plus diplômées sont mieux préparées pour réussir leur migration et s'insérer dans le tissu économique canadien. À cela s'ajoutent la diversité des origines géographiques de ces migrants issue du monde sinisé et le poids des événements historiques. Le fossé est grand entre les migrants anciens résidents de la colonie britanniques de Hong Kong, les étudiants réfugiés de l'après Tienanmen et les dernières générations de diplômés de la Chine de l'ouverture économique. La Chinatown ne revêt pas la même valeur ni la même signification pour tous. Le quartier est donc délaissé par les nouveaux migrants aux statuts économiques plus confortables qui privilégient davantage les boutiques chinoises dispersées dans centres commerciaux de banlieues. Mais ce lieu reste emblématique pour les immigrants individuels les moins favorisés, chinois et asiatiques en général, tout comme pour les jeunes Chinois nés sur le sol canadien qui aiment s'y retrouver, renouer avec leur culture lors de grands événements.

17. «Charting on future», The Ottawa Citizen, 16 octobre 2003. 


\section{Conclusion}

L'histoire migratoire du Canada a été marquée à la fin du XXe siècle par l'ampleur nouvelle des migrations issues du monde asiatique et notamment de la Chine. D'ailleurs depuis 1996, la langue maternelle chinoise remplace l'italien au premier rang des langues maternelles non officielles les plus fréquemment déclarées au Canada : c'est la langue maternelle de 2,9\% de la population canadienne (soit 849190 en 2001).

L'analyse spatiale met en évidence l'évolution des stratégies territoriales des immigrants qui révèlent des polarisations majeures au profit des régions urbaines les plus riches et les plus dynamiques du pays : tout d'abord Toronto et son aire métropolitaine, puis Vancouver. Ensuite il est à noter une modeste diffusion vers les autres centres urbains du pays que l'immigrant ne perçoit qu'en terme de périphérique où son installation est alors conçue comme une étape dans le cadre de sa stratégie professionnelle qui doit le conduire inéluctablement vers les régions métropolitaines les plus dynamiques.

Les choix géographiques des immigrants chinois rappellent que les causes de l'immigration chinoise au Canada n'ont pas tellement changé. La motivation principale reste aujourd'hui encore la recherche de meilleures conditions de vie et de salaire, pour tous les migrants quel que soit leur niveau de qualification, y compris les derniers immigrants dont le niveau de scolarité est meilleur ${ }^{18}$.

Plus que jamais les immigrants chinois anciens et nouveaux forment bien une communauté plurielle où les différences sociales, culturelles et économiques sont importantes. Les nouvelles générations plus diplômées sont mieux préparées pour réussir leur migration et s’insérer dans le tissu économique canadien. À cela s'ajoutent la diversité des origines géographiques de ces migrants issue du monde sinisé et le poids des événements historiques. Le fossé est grand entre les migrants anciens résidents de la colonie britanniques de Hong Kong, les étudiants réfugiés de l'après Tienanmen et les dernières générations de diplômés de la Chine de l'ouverture économique. La Chinatown ne revêt pas la même valeur ni la même signification pour tous. Le quartier est donc délaissé par les nouveaux migrants aux statuts économiques plus confortables qui privilégient davantage les boutiques chinoises dispersées dans centres commerciaux de banlieues. Mais ce lieu reste emblématique pour les immigrants individuels les moins favorisés, chinois et asiatiques en général. Tout comme pour les jeunes chinois nés sur le sol canadien qui aiment s'y retrouver, renouer avec leur culture lors de grands événements.

Les Sino-canadiens constituent une catégorie de population de plus en plus hétérogène, composée de sous communautés aux identités et profils socio-économiques spécifiques, issus de contextes historiques et des origines géographiques diverses comme le souligne l'état de la communauté chinoise d'Ottawa qui s'est graduellement désintégrée en diverses sous-communautés. Dans ce contexte, l'espace de vie des migrants s'individualise davantage et l'image emblématique des Chinatowns d'aujourd'hui est révélatrice de ces transformations : l'enclave urbaine d'hier est désormais un lieu commercial animé, un lieu culturel, un lieu de rencontre pour les Asiatiques et les citadins en général tandis que les nouveaux migrants chinois s'installent dans les banlieues canadiennes.

\section{Bibliographie}

FAnG (D.), Brown (D.), 1999. - « Geographic Mobility of the Foreign-Born Chinese in Large Metropolises, 1985-1990 », International Migration Review, vol. 33, n 1, p. 137-155.

18. Environ $34 \%$ des immigrants récents âgés de 25 à 44 ans avaient terminé leurs études universitaires, contre $19 \%$ de la population de la même classe d'âge née au Canada. 
GitTings (J.), 1989. - China Changes Face: The Road from Revolution 1949-1989, Oxford, Oxford University Press, $234 \mathrm{p}$.

INFRASTRUCTURE CANADA, 2005. - Note de recherche: Bref aperçu de la recherche sur les quartiers au Canada, Division recherche et analyse, Ottawa, 4 p. [http://www.infrastructure.gc.ca/research-recherche/rresul/rn/ documents/rn05_f.pdf].

Keung (N.), 2004. - Ethnic mini-cities on rise, StatsCan study finds; Immigrants settle in enclaves - Concerns raised about isolation, Toronto Star, Mar 10, p. A.01.

LAI (D. C.), 2003. - «From Downtown Slums to Suburban Malls : Chinese Migration and Settlement in Canada », dans Laurence (J. C.), Cartier (C.) (ed.), The Chinese Diaspora - Space, Place, Mobility, and Identity, p. 311-336.

—, 1988. - Chinatowns : Towns Within Cities In Canada, Vancouver, University of British Colombia Press, 285 p.

Li (P. S.), 1988. - The Chinese In Canada, Toronto, Oxford University Press, 134 p.

Li (Q.), 2000. - Ethnic minority churches: the cases of the Canadian Chinese Christian churches in Ottawa, Thesis, University of Ottawa, 333 p.

Liu (X.-F.), 1997. - « Refugee Flow or Brain? The Humanitarian Policy and Post-Tiananmen Mainland Chinese Immigration to Canada », International Journal of Population Geography, vol. 3, p. 15-29.

Lo (L.), WANG (S.), 1997. - « Settlement patterns of Toronto's Chinese immigrants : convergence or divergence? ", Canadian Journal of Regional Science, vol. 20, p.49-72.

O’Neill (J.), 2003. - « Crossing Canada's 'racial divide': Series : Our Town : In Living Colour », The Ottawa Citizen, Ottawa, october 18, p. B.1.Fro.

SHI (Y.), 2004. - «The Impact of Canada's New Immigration Act on Chinese Independent Immigrants », Canadian Journal of Urban Research, vol. 13, n 1, p. 140-154.

Statistique Canada, Hou (F.), 2004. - Document de recherche: Immigration récente et formation de quartiers de minorités visibles dans les grandes villes canadiennes, Division de l'analyse des entreprises et du marché du travail, no 11F0019MIF au catalogue, n² 221, Ottawa [http://www.statcan.ca/francais/research/ 11 f0019mif/11f0019mif2004221.pdf].

Statistique CANADA, 2001. - [http://www.statcan.ca/francais/Pgdb/defdemo25_f.htm].

Wickberg (P.) (éd.), 1984. - Histoire des peuples du Canada. « de la Chine au Canada»: Histoire des communautés chinoises au Canada, Ottawa, Centre d'édition du gouvernement du Canada «ministère des Approvisionnements et Services » Canada, 287 p.

Cet article a été reçu le 14 juillet 2005 et définitivement accepté le 10 avril 2006. 